\title{
Diabetes Mellitus y Disfunción Endotelial
}

\author{
Diabetes mellitus and Endothelial Dysfunction
}

\author{
César Delgado-Butrón ${ }^{1 \dagger}$, César Delgado-Torres ${ }^{2}$, Leonidas Delgado-Torres $^{3}$
}

\section{Resumen}

La Disfunción Endotelial (DE) en la diabetes resulta de múltiples factores metabólicos tributarios de la glucotoxicidad, lipotoxicidad, resistencia a la insulina y de la interacción recíproca entre ellos. Hay un número de alteraciones funcionales consiguiente a la DE, como: proliferación de las células endoteliales, anormalidades del flujo y permeabilidad vascular, angiogénesis patológica, disminución de la fibrinolisis y oclusión vascular, incremento de la expresión de genes pro inflamatorios y variados efectos dependientes del aumento de especies de oxígeno reactivo (ROS) y del estrés oxidativo. Estos y otros efectos no bien conocidos, hacen de la DE un componente relevante y clave en la patogénesis de la micro y macrovasculoptía diabética. Las complicaciones microvasculares en la retina, glomérulo y vaso nervorum en la diabetes mellitus, implican función anormal de la célula endotelial, caracterizada por cambios vasculares, producción de factores de crecimiento y citokinas, activación de genes proinflamatorios, depósito de matriz extracelular y otros, lo cual puede ser considerado como la primera etapa de la progresión de la microangiopatía diabética. En cuanto a las complicaciones macrovasculares es importante resaltar que la DE precede a la aterosclerosis.

Palabras clave: Disfunción Endotelial, Diabetes Mellitus.

\section{Abstract}

The Endothelial Dysfunction (ED) in diabetes results from a variety of metabolic factors wich influence the glucotoxicity, lipotoxiciy, insulin resistance and the reciprocal interaction between them. There are a number of functional alterations following to ED, as: active endothelial cell proliferation, abnormalities in vascular flux and permeability, pathologic angiogenesis, decrease of the fibrinolysis, vascular occlusion, increase proinflamatory gene expression and multiple effects dependent on the increment of reactive oxygen species (ROS) and the oxidative stress. These and other poorly understood effects make the ED a key and relevant component in the pathogenesis of diabetic micro and macrovasculopahy. The microvascular complications in retina, glomerulus and vasa nervorum in the diabetes mellitus implicate an abnormal function of the endothelial cell, characterized by vascular changes, production of growth factors and cytokins, activation of proinflamatory genes, deposition of extracelular matrix and others, wich can be considered like the first step in the progression of diabetic micrangiopathy. As far as the macrovascular complications, it is important to emphasize that ED precedes atheroesclerosis.

Keywords: Endothelial Dysfunction, DiabetesMellitus.

\section{Introducción}

Las complicaciones micro y macrovasculares de la diabetes es el resultado de un ambiente adverso vascular generado, a su vez, por el disturbio metabólico consecuente al estado de hiperglicemia. Las complicaciones microvasculares que afectan a la retina, glomérulo y vasos nervorum son específicas de la diabetes y clínicamente se expresan en la retinopatía, nefropatía y neuropatía, que son las causas principales de ceguera y de insuficiencia renal terminal, entre otros. Por su lado, las complicaciones macrovasculares, no específicas de la diabetes, dan cuenta de la cardiopatía coronaria, accidente cerebro vascular y enfermedad vascular periférica, cuya frecuencia es marcadamente elevada en comparación a la población no diabética.
El proceso de disfunción endotelial, es un evento clave en la patogénesis de la micro y macrovasculopatía diabética y constituye la primera etapa de la secuencia de eventos adversos que lleva al daño aterosclerótico.

En el presente artículo revisamos el eslabón entre las anormalidades metabólicas subyacentes a la hiperglicemia y la disfunción endotelial.

\section{Rol Fisiológico del Endotelio}

El endotelio es el órgano más grande del cuerpo humano y aparte de proporcionar una barrera física entre la

${ }^{1}$ Profesor Emérito de la Universidad Nacional de San Agustín de Arequipa. ${ }^{2}$ Médico Endocrinólogo: Asistente del Servicio de Endocrinología del Hospital Nacional EsSalud, Arequipa. Profesor auxiliar de la Universidad Nacional de San Agustín de Arequipa. ${ }^{3}$ Médico Patólogo Clínico: Investigador del Centro Endocrinológico Delgado, Arequipa. 
pared vascular y el lumen, promueve múltiples funciones, principalmente a travésde la producción de óxido nítrico (NO) generado por la acción de la sintasa de óxido nítrico endotelial (eNOS). Se han identificado tres isoformas de NOS: eNOS (NOS endotelial), nNOS (NOS neuronal) e iNOS (NOS inducible). eNOS y nNOS se les denomina también cNOS (NOS constitutiva) ${ }^{(1)}$.

Entre las funciones del endotelio se tienen, que: ${ }^{(2,3)}$ Modula el tono vascular mediante la secreción de vasodilatadores: $\mathrm{NO}$, prostaciclina $\mathrm{I}_{2}\left(\mathrm{PGI}_{2}\right)$, factor hiperpolarizante derivado del endotelio (EDHF) y péptido natriurético tipo $\mathrm{C}$, que guardan equilibrio con la secreción de vasoconstrictores: endotelina -1 (ET-1), angiotensina II (AT II), prostaglandina $\mathrm{H}_{2}\left(\mathrm{PG}_{2}\right)$, tromboxano $\mathrm{A}_{2}\left(\mathrm{TXA}_{2}\right)$ y especies de oxígeno reactivo (ROS).

- Asegura el adecuado flujo sanguíneo.

-Atenúa la producción de citoquinas inflamatorias.

- Limita el reclutamiento de leucocitos.

- Disminuye la expresión de VCAM (molécula de adhesión de células vasculares).

- Inhibe la proliferación de VSMC (células del músculo liso vascular).

- Regula la agregación plaquetaria, coagulación y fibrinolisis.

-Inhibe la apoptosis.

- Reduce la adhesión de monocitos a la pared vascular.

- Regula la permeabilidad y vascular.

\section{Disfunción Endotelial (DE)}

Drexler $\mathrm{H}^{(4)}$, conceptúa a la disfunción endotelial como "un fenotipo endotelial mal adaptado, caracterizado por biodisponibilidad de NO reducido, estrés oxidativo incrementado, expresión elevada de factores protrombóticos y proinflamatorios y vasoreactividad anormal", es decir, que el endotelio pierde sus propiedades fisiológicas: tendencia a promover, fibrinolisis y antiagregación plaquetaria. La DE precede o está asociada con diversas patologías como hipertensión arterial, enfermedad coronaria, falla renal crónica y a condiciones de resistencia a la insulina (diabetes, obesidad, síndrome metabólico y otros estados patológicos).

\section{Anormalidades Metabólicas y Disfunción Endotelial en la Diabetes Mellitus ${ }^{(5-7)}$}

Las observaciones en el modelo tanto animal y clínico indican que la hiperglicemia crónica es el factor iniciador central para todos los tipos de enfermedad microvascular diabética (EMD). La duración y magnitud de la hiperglicemia se correlacionan marcadamente con el grado y velocidad de progresión de la EMD. En apoyo de lo anterior están los resultados $\mathrm{DCCT}^{(8)}$ y UKPDS ${ }^{(9)}$, que encontraron reducción de la incidencia de las complicaciones microvasculares con terapia intensiva de insulina. Otra característica de las células endoteliales es que desarrollan hiperglicemia intracelular debido a que ellas no pueden "down regulate" el transporte de glucosa cuando son expuestas a hiperglicemia extracelular, lo que difiere de muchas otras células ${ }^{(\uparrow)}$.

Otro concepto que abala la influencia de la hiperglicemia es la llamada memoria hiperglicémica, definida como la persistencia o progresión de las alteraciones microvasculares por la hiperglicemia, durante períodos subsecuentes de homeostasis de glucosa normal ${ }^{(11)}$.

La hiperglicemia, como se describirá a continuación, genera metabolitos tóxicos que ejercen efectos adversos sobre la función endotelial, a saber: estimulación de la proteína kinasa $\mathrm{C}$ (PKC), incremento de productos finales de glicación avanzada (AGEs), formación de sorbitol y producción aumentada de $\operatorname{ROS}^{(12,13)}$. La activación de estas vías promueven incremento de estrés oxidativo vascular, inflamación, apoptosis, aterogénesis y función endotelial alterada, lo que finalmente conducirá a las complicaciones crónicas de la diabetes, tanto micro como macrovasculares.

\section{Hiperglicemia y Activación de la Proteína Kinasa C (PKC)}

El estado de hiperglicemia promueve la activación de PKC que incluye 11 isoformas, 9 de las cuales son activadas por la producción incrementada de diacilglicerol(DAG) a través de la glicólisis. El incremento de PKC causa una variedad de alteraciones proaterogénicas: ${ }^{(12-15)}$

\footnotetext{
- Reducción del flujo sanguíneo renal y retinal por disminución de NO y aumento de ET -1.

-Aumento de la permeabilidad y angiogénesis vascular por incremento del factor de crecimiento endotelial vasular(VEGF).

-Acumulación de matriz proteica extracelular (incremento de colágeno y fibronectina). - Inhibición de la fibrinolisis por aumento del inhibidor del activador de plasminógeno tipo -1 (PAI-1). -Expresión de genes proinflamatorios por aumento del factor nuclear Kappa B (NF-kB).

-Activación de varias oxidasas dependientes de NADPH, incrementando ROS. (Figura 1).
}

\section{Hiperglicemia y Formación de Sorbitol (Vía del Polyol)}

El metabolismo de la glucosa por la vía del polyol en sujetos no diabéticos ocurre en un pequeño porcentaje, sin embargo, en la situación de hiperglicemia del paciente diabético, la conversión enzimática a sorbitol se incrementa (Figura 2). La aldosareductasa reduce aldehidos tóxicos generados por ROS a alcoholes inactivos y la glucosa intracelular incrementada a sorbitol, luego éste es oxidado a fructosa por acción de la enzima sorbitol dehidrogenasa y como cofactor el dinucleótido adenina nicotinamida (NAD+ reducido a NADH) ${ }^{(16)}$. La 


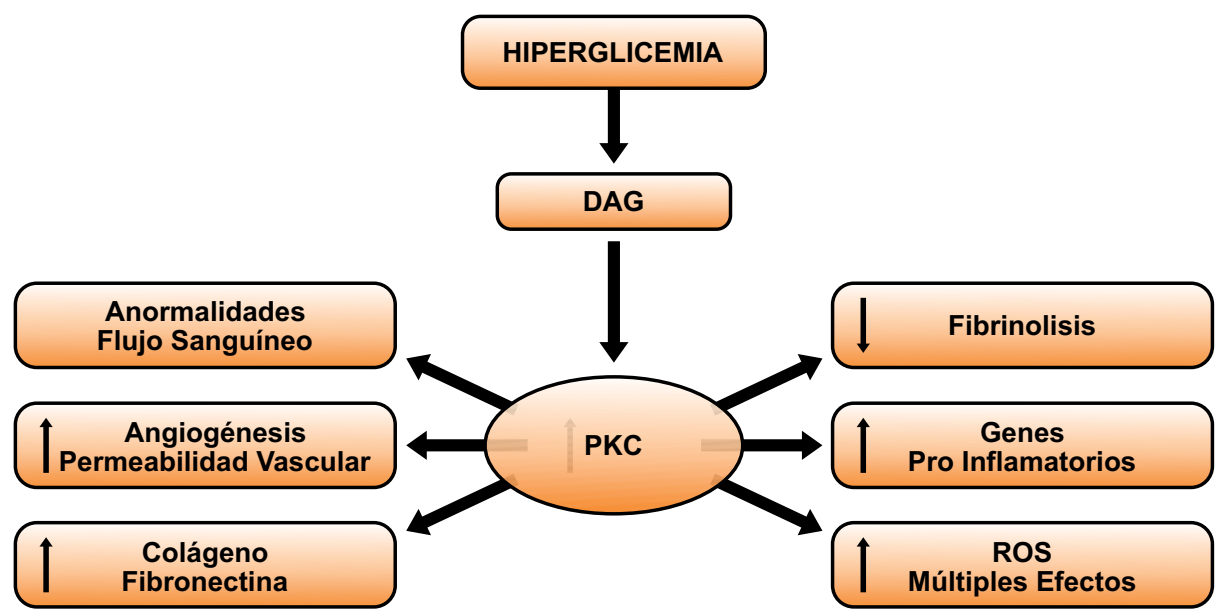

Figura 1. Hiperglicemia y actividad de la proteína Kinasa C (PKC). DAG: Diacilglicerol.

utilización de la glucosa por esta vía varía de acuerdo a la especie, tejido, entre otros; de modo que las complicaciones diabéticas por la vía del polyol-sorbitol depende de muchas variables.

El mecanismo de acción de la vía polyol-sorbitol como posible causa del deterioro ocasionado por la hiperglicemia no está bien dilucidado. Se han propuesto diversas acciones del sorbitol, como que: provoca daño osmótico, inducción de estrés oxidativo, disminución de la actividad de la bomba de sodio potasio dependiente de la adenosina trifosfatasa $\left(\mathrm{Na}^{+} \mathrm{K}^{+}\right.$ATPasa), aumento de la relación NADH/NAD+ citosólico y disminución de NADPH citosólico. Cada una de estas hipótesis no aportan evidencias convincentes ${ }^{(17)}$.

La oxidación de sorbitol por NAD+ produce inhibición de la actividad de la enzima giceroaldehido 3 fosfato dehidrogensasa (GADPH), dando lugar a que la glucosa se desvíe de la glicólisis a otras vías de utilización, generando PKC y AGEs ${ }^{(\uparrow 2,13)}$.

La formación de sorbitol a partir de glucosa consume nicotinamida adenina dinucleótido fosfato reducido (NADPH) $\mathrm{y}$, a su vez, disminuye la generación de glutation reducido, lo cual exacerba el estrés oxidativo intracelular. El NO mantiene a la enzima aldosa reductasa en forma inactiva, situación que se pierde en la diabetes $^{(12)}$.

En estudios en animales, la inhibición de la aldosa reductasa previene algunas anormalidades en la retinopatía, nefropatía y neuropatía diabética ${ }^{(13)}$.

En pacientes con neuropatía diabética la inhibición de la aldosa reductasa con zenarestat produjo buenos resultados (18).

\section{Hiperglicemia y Formación de Productos Finales de Glicación Avanzada (AGEs) ${ }^{(12,19-22)}$}

Actualmente se acepta que la hiperglicemia intracelular sea el inductor primario en la formación tanto de AGEs intra y extracelulares. Actúan a través de sus receptores (RAGE). Tres vías conducen a la formación de AGEs: (Figura 3).

- Vía glioxal resultante de la autooxidación intracelular de la glucosa.

- Vía 3-deoxiglucosona a partir de la descomposición del producto Amadoriy

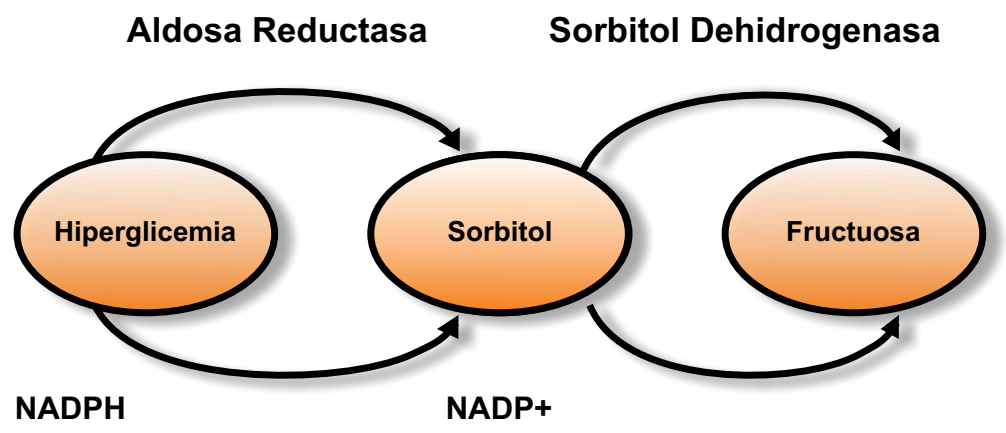

Figura 2. Hiperglicemia: Vía Polyol. 


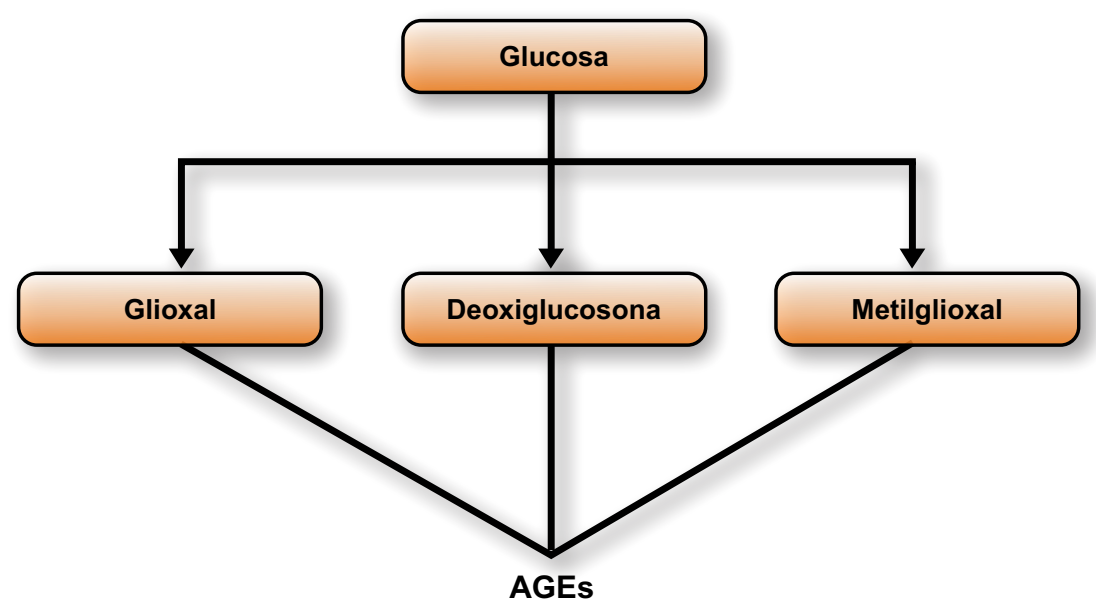

Figura 3. Precursores de la formación de los productos finales de la glicación avanzada (AGEs).

-La Vía metilglioxal que se origina por fragmentación del gliceroaldehido 3 fosfato durante la glicolisis.

En las células endoteliales la vía metilglioxal es el precursor más importante de los AGEs.

Los precursores de AGEs dañan a las células endoteliales por 3 mecanismos: (Figura 4 ).

- Modifican las proteínas intracelulares, consecuentemente alteran su función.

-Modifican los componentes de la matriz extracelular e in- teractúan anormalmente con otros componentes y receptode la misma matriz.

-Alteran proteínas plasmáticas que al unirse a receptores AGEs sobre células como macrófagos inducen la producción de ROS.

Los AGES han mostrado que directamente injurian a las células beta del páncreas.

Los precursores de AGEs "up-regulation" NADPH oxidasa y, a su vez, incrementa el estrés oxidativo en células endoteliales humanas, un sustento molecular de la vasculopatía diabética.

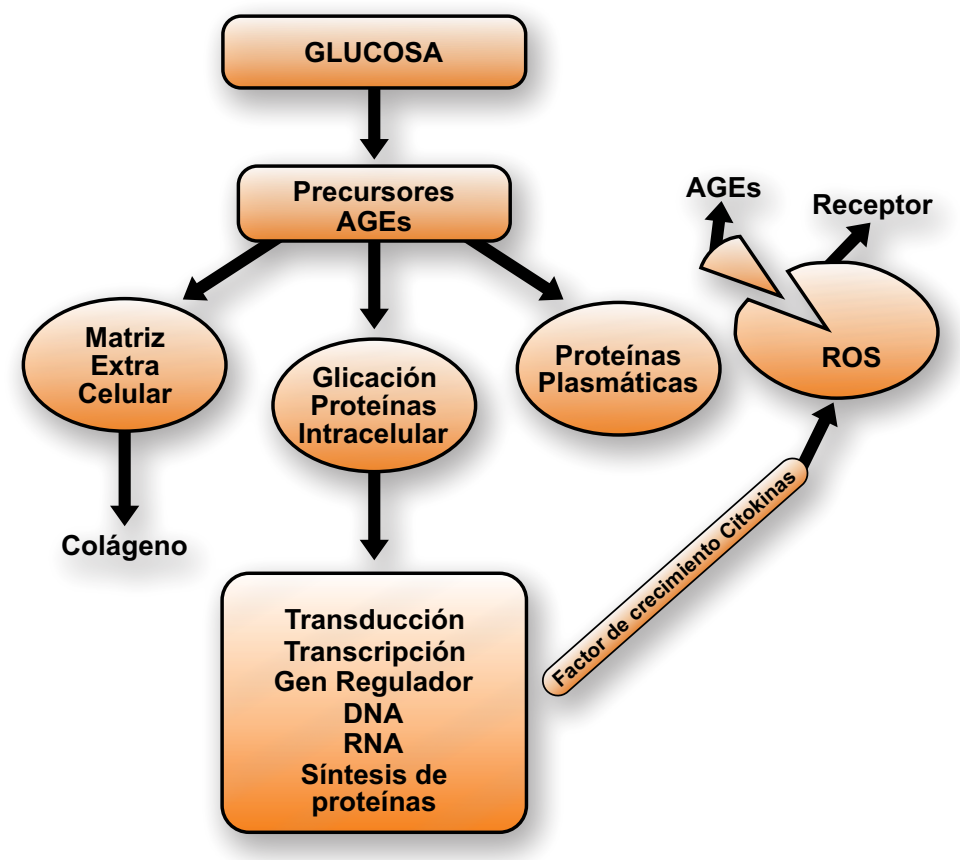

Figura 4. Daño celular por los precursores de AGEs. 
En la retinopatíadiabética humana, los AGEs causan incremento de la permeabilidad capilar, contribuyendo tanto en el edema macular y neovascularización de la retina por aumento de la expresión del VEGF.

En la fase temprana de la nefropatíadiabética los AGEs favorecen la hiperfiltración y microalbuminuria por estímulo de la secreción de VEGF y de la proteína quimotáctica de monocito 1 (MCP1). Además, los AGEs contribuyen a las lesiones más avanzadas de la nefropatíadiabética promoviendo apoptosis de las células mesangiales del glomérulo.

Los efectos deletereos de los AGEs asociados a la hiperglicemia no se producen bloqueando los RAGEs, lo que da lugar a una inhibición del desarrollo de la vasculopatía.
Hiperglicemia y Activación de la

Vía de la Hexosamina ${ }^{(12,13,23)}$

A parte de las 3 vías descritas de la hiperglicemia como causantes de los trastornos metabólicos que llevan a las complicaciones diabéticas, se suma una cuarta en que la glucosa se desvía a la vía de la hexosamina, la cual sumariamente consiste en que: la fructosa 6 fosfato procedente de la glicolisis, por acción de la glutamina fructosa 6-fosfato amidotransferasa (GFAT), es convertida a glucosamina-6fosfato (GLc-6-P) como sustrato de uridin-difosfato $\mathrm{N}$-acetil glucosamina (UDP-GLcNAc). A través de estos intermediarios se producen cambios en los genes de transcripción, incrementando la producción de factores de promoción de las complicaciones diabéticas, como el inhibidor del activador de

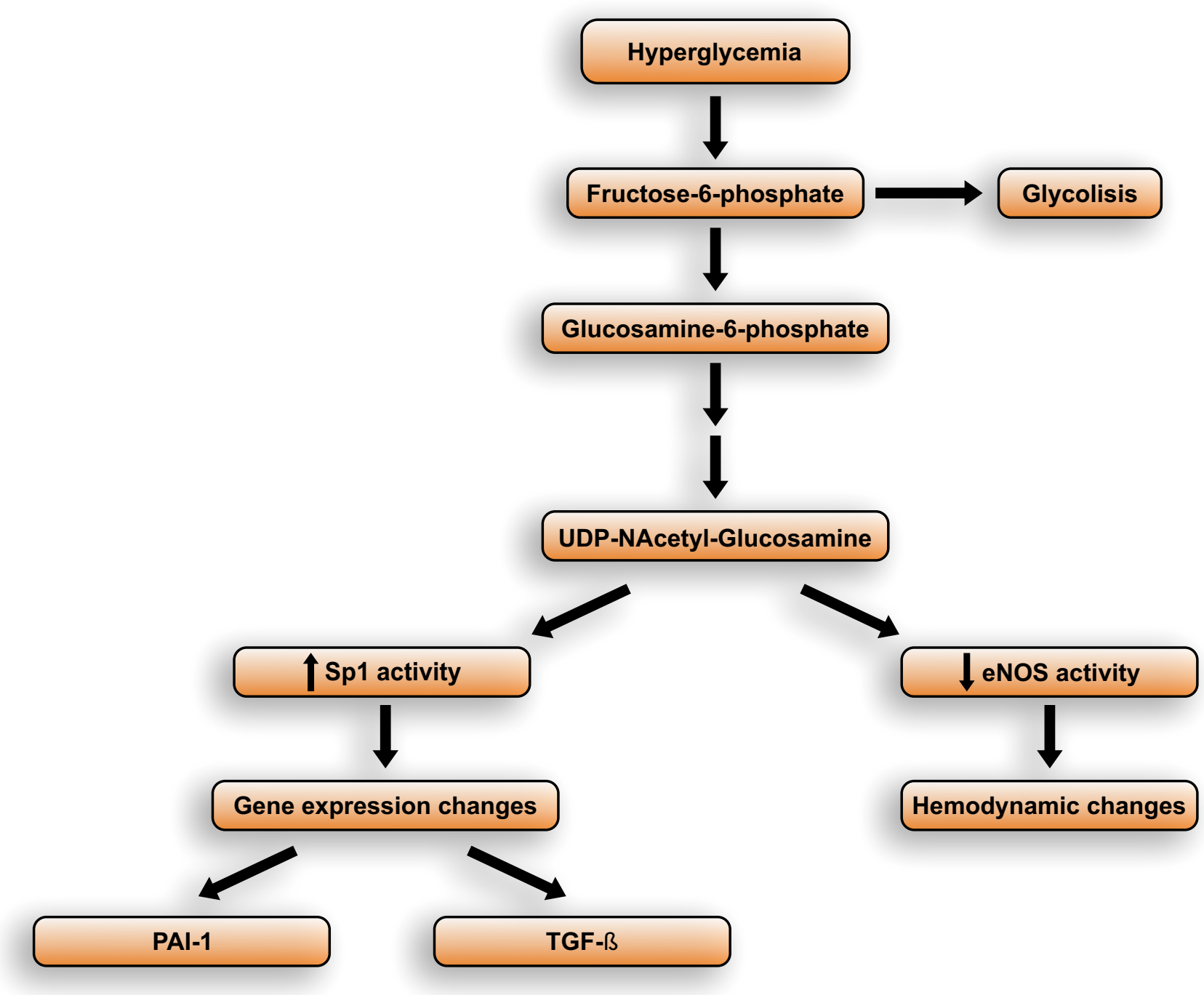

Figura 1. Hiperglicemia y activación de la vía de la Hexosamina.

Sp1 : Factor de transcripción. Sp1; eNOS : Sintetasa de óxido nítrico endotelial; $\mathbf{P A I}=1:$ Inhibidor del activador del plasminógeno $1 ;$ TGF- $\beta$ : Factor de crecimiento transformante $\beta$. 
plasminógeno I (PAI-1) y el factor de crecimiento de transformación beta (TGF- 3 ) (Figura 5).

La hiperglicemia ha mostrado un incremento de 2.4 veces la actividad de la vía de la hexosamina de las células endoteliales aórticas, y que esta vía aumenta la activación de las metaloproteasas (MMP2 y MMP9) en las células endoteliales de la arteria coronaria humana y en las placas de la carótida de pacientes con diabetes mellitus tipo 2 .

La vía de la hexosamina también puede causar múltiples cambios en la expresión del gen y en la función proteica, como consecuencia de una glicosilación alterada de $\mathrm{N}$-acetil glucosamina, contribuyendo en la patogénesis de las complicaciones diabéticas.

\section{Vía Común Final de la Hiperglicemia: \\ Incremento de Especies de Oxígeno \\ Reactivo(ROS)}

También conocido como el "mecanismo de unificación del daño celular inducido por la hiperglicemia"(24). Los 4 mecanismos patogénicos desencadenados por la hiperglicemia, ya referidos, confluyen y se expresan en un único proceso: la sobreproducción de ROS, lo que finalmente conduce a la disfunción vascular. Dos fuentes de ROS estimulados por la hiperglicemia son reconocidas: la generación de NADH y piruvato en el proceso glicolítico citoplasmático y la oxidación del piruvato mitocondrial en el ciclo de Krebs, ambas fuentes lo realizan a través de la cadena de transportes de electrones en la mitocondria. El incremento de ROS por la hiperglicemia es inhibida por la dismutasa superóxido de manganeso (MnSOD)-enzima antioxidante mitocondrial- así como por agentes que desacoplan la fosforilacion oxidativa; por consiguiente, evita la activación de la vía del poliol, la formación de AGEs, de PKC y de la vía de la hexosamina; contrariamente la activación de las 4 vías anteriores convergen en paralelo con la formación de ROS secundaria a la hiperglicemia ${ }^{(12)}$.

El mecanismo por el cual el superóxido mitocondrial inducido por la hiperglicemia activa los cuatro procesos ya señalados, se debería a que el exceso de superóxido inhibe parcialmente la enzima glicolítica, gliceroaldehido-3-fosfato dehidrogenasa (GAPDH); en consecuencia los compuestos intermediarios previos a este bloqueo se acumulan, así el incremento del gliceroaldehido-3-fosfato aumenta la síntesis de metilglyoxal y diacilglicerol, precursores básicos para la formación de AGEs y PKC; en tanto que la glucosa y fructosa incrementan la vía de la hexosamina y del poliol, respectivamente $^{(25)}$.

Otros efectos del incremento de ROS que conducen a la disfunción vascular generada por la hiperglicemia, son: el promover directamente la remodelación y apoptosis vascular, como se observa en las células endoteliales de la aorta humana; estimular la metaloproteinasa de la matrix macular (MMP) en las células endoteliales de la aorta bovina, lo cual reduce la disponibilidad del NO. El volumen del glicocalix-capa de proteoglicanos que cubren el endotelio- también está reducido por la hiperglicemia aguda y exacerba la disfunción endotelial en sujetos humanos sanos ${ }^{(20,26)}$.
ROS directamente inactiva 2 enzimas antiaterogénicas: sintasa de prostaciclina y sintasa de óxido nítrico endotelial.

En resumen, la interrelación entre hiperglicemia-con sus metabolitos tóxicos de las 4 vías -y la formación de ROS son partícipes importantes en la disfunción endotelial y estos cambios metabólicos se expresan clínicamente en las complicaciones vasculares crónicas, tanto micro como macroangiopáticas; por ello, la mejor manera de detener esta secuencia de eventos es un buen control glicémico del diabético con los medios terapéuticos actuales.

\section{Interrelaciones entre Disfunción Endotelial y Resistencia a la Insulina}

Existe una relación recíproca entre el desarrollo de la DE y resistencia a la insulina (RI), de modo que la DE y RE frecuentemente coexisten ${ }^{(27)}$. Algunos estudios sugieren que la $\mathrm{DE}$ predice la incidencia de diabetes mellitus ${ }^{(28,29)}$. Biomarcadores en general y marcadores hemostáticos de DE incrementan el riesgo de diabetes mellitus tipo 2, independientemente de otros factores de riesgo para la diabetes (obesidad, RI, entre otros). También niveles elevados de moléculas de adhesión endotelial como: E-selectina e ICAM-I, se asocian con incrementado riesgo de desarrollo de diabetes ${ }^{(27)}$. Hijos no diabéticos de padres diabéticos tienen tanto RI y $\mathrm{DE}^{(30)}$. Estos y otros estudios apoyan un rol causal potencial de la DE en la RI. De otra parte, la RI se acompaña de alteraciones vasculares características de la DE, como la incapacidad para la vasodilatación. En la figura 6 se observa que la RI afecta por diversas alteraciones metabólicas la cascada de la señalización de la insulina, vía fosfatidilinositol 3-kinasa (PI3K) y proteína Kinasa $\mathrm{B}(\mathrm{AKt})$, mientras que la vía mitógeno activada por la proteína kinasa (MAPK) no es afectada. El inbalance entre PI3K y MAPK lleva a modificaciones vasculares y metabólicas, como consecuencia de la DE y de la RI, produciendo finalmente aumento de la endotelina 1 y disminución de NO y GLUT4, con lo que predomina la vasoconstricción y disminuye la captación de glucosa en los tejidos blancos ${ }^{(31,32)}$.

\section{Rol de los Ácidos Grasos Libres en la Disfunción Endotelial Asociada a la Diabetes Mellitus}

Otra consecuencia de la RI es que causa aumento de la liberación de los ácidos grasos libres (FFA) desde los adipocitos e incrementan la oxidación de éstos en la mitocondria de la célula endotelial, con lo que se activa las vías aterogénicas e inhiben las enzimas antiaterogénicas.

La oxidación de FFA, al igual que la oxidación de la glucosa en el ciclo de Krebs, genera exceso de ROS y mediante el mismo mecanismo, como ocurre con la hiperglicemia, activan las vías de la glucotoxicidad: PKC, AGEs, hexosamina $\mathrm{y}$ factor nuclear $\mathrm{KB}$, todo ello favorece los procesos aterogénicos.

De otro lado, el exceso de FFA está implicado en la patogénesis de la RI. Al igual que la glucotoxicidad, la lipotoxicidad inducida por FFA puede promover DE por varios mecanismos: reduce la disponibilidad de NO, aumenta el estrés 


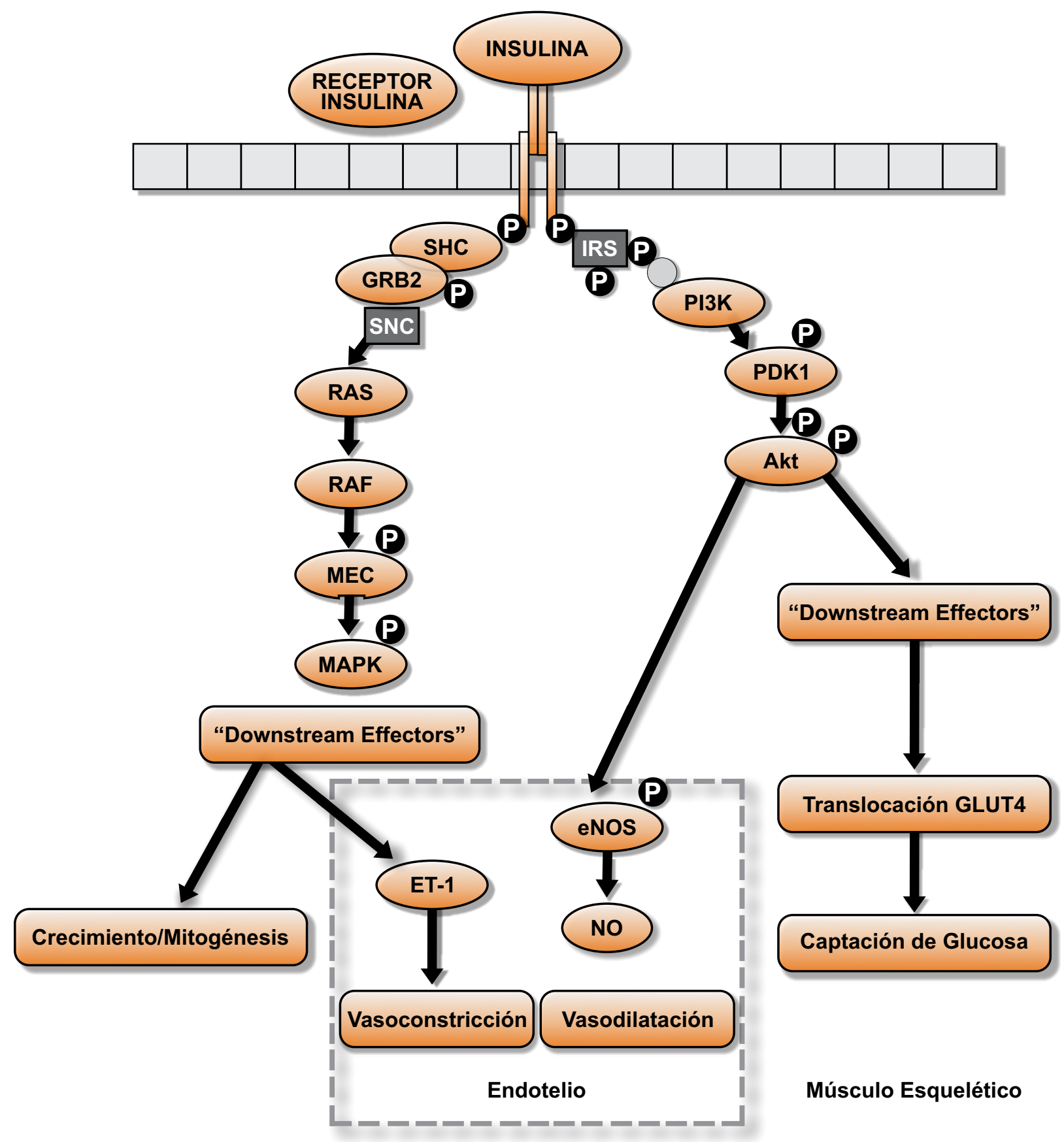

Figura 5. Resistencia a la insulina y alteración de la cascada de la señalización de la insulina.

oxidativo vascular, inhibe la fosforilacion de IRS-1, incrementa la desintegrina y metaloproteinasa, activa la proliferación y migración de células y permeabilidad endotelial, aumenta la adhesión de monocitos al endotelio, incrementa la respuesta inflamatoria e induce la apoptosis endotelial ${ }^{(20,32,33)}$.

Los efectos de los FFA sobre la función endotelial han sido demostrados en modelos animales in vitro e in vivo y en humanos; así en el conejo, la incubación in vitro con FFA desmejora la función endotelial en anillos aórticos aislados, acompañado por niveles de NO reducido y estrés oxidativo aumentado. En humanos sanos expuestos a FFA elevados incrementa marcadores plasmáticos de actividad endotelial, tales como: ICAM-1, VCAM-1 y selectina E soluble y aumenta los niveles plasmáticos de miel o peroxidasa (MPO) y del inhibidor del activador plasminógeno tisular ${ }^{(20,34)}$. Por consiguiente, la glucotoxicidad, lipotoxicidad e inflamación contribuyen al desarrollo de la RI y paralelamente de la DE. 
Referencias bibliográficas

1.Avogaro A, Fadini GP, Gallo A, et al. Endothelial dysfunction in type 2 diabetes mellitus. Nutrition, Metabolism and Cardiovascu- lar Diseases 2006;16:539.

2.Hartge MM, Pharm BP, Kintscher U. Endothelial dysfunction and its role in diabetic vascular disease. Endocrinol Metab Clin NAm 2006;35: 551.

3.Muniyappa R, Iantorno M, Quon MJ. An integrated view of insulin resistance and endothelial dysfunction. Endocrinal Metab Clin N Am 2008:37:685.

4.Drexler H. Endothelial dysfunction: clinical implications. Prog Cardiovasc Dis 1997;39(4):287.

5.Monnink SH, van Haelst PL, van Boven AJ, et al. Endothelial dysfunction in patients with coronary artery disease: a comparison of three frequently reported tests. J Investig Med 2002;50(1):19.

6. Bolton CH, Downs LG, Victory JG, et al. Endothelial dysfunction in chronic renal failure: roles of lipoprotein oxidation and pro inflammatory cytokines. Nephrol Dial Transplant 2001;16(6):1189.

7.Kim JA, Montagnani M, Koh KK, et al. Reciprocal relationships between insulin resistance and endothelial dysfunction: molecular and pathophysiological mechanisms. Circulation 2006;113(15):1888.

8.DCCT Research Group. The effect of intensive treatment of diabetes on the development and progression of long-term complications in insulin dependent diabetes mellitus. N Engl J Med 1993;329:977.

9.UK. Prospective Diabetes Study (UKPDS) Group. Intensive blood glucose control with sulphonylureas or insulin compared with conventional treatment and risk of complications in patients with type 2 diabetes. Lancet 1998;352:837.

10.Kaiser N, Freener EP, Boukobza-Vardi N, et al. Differential regulation of glucose transport and transporters by glucose in vascular endothelial and smooth muscle cells. Diabetes 1993;42:80.

11.Engerman RL, Kern TS. Progression of incipient diabetic retinopathy during good glycemic control. Diabetes 1987;36:808.

12. Brownlee M, Aiello LLP, Cooper ME, et al. Complications of diabetes mellitus. In: Melmed S, Polonsky KS, Larsen PR, Kronenberg HM, eds. Williams Textbook of Endocrinology. 12th edition. ELSEVIER Saunders. Philadelphia 2011;1462.

13. He Z, King GL. Microvascular complications of diabetes. Endocrinal Metab Clin NAm 2004;33:2015.

14. Willams B, Gallacher B, Patel $\mathbf{H}$, et al. Glucose induced protein kinase $\mathrm{C}$ activation regulates vascular permeability factor mRNA expression and peptide production by human vascular smooth muscle cells in vitro. Diabetes 1997;46:1497.

15. Geraldes P, King GL. Activation of protein kinase $C$ isoforms and its impact on diabetic complications. Cir Res 2010;106:1319.

16. Sheetz MJ, Kin GL. Molecular understanding of hyperglycemia's adverse effects for diabetic complications. JAMA 2002;288:2579.

17. Brownlee M. Biochemistry and molecular cell biology of diabetic complications. Nature 2001;414:813.

18. Greene DA, Arezzo JC, Brown MB. Effect of aldose reductase inhibition on nerve conduction and morphometry in diabetic neuropathy. Zenarestat Study Group. Neurology 1999;53:580.
19.Vlassara H, Striker GE. Advanced glycation endproducts in diabetes and diabetic complications. Endocrinal Metab Clin NAm 2013;42:697.

20.Zhang H, Dellsperger KC, Zhang C. The link between metabolic abnormalities and endothelial dysfunction in type 2 diabetes: an update. Basic Res Cardiol 2012;107;237.

21.Rodino- Janeiro BK, Gonzalez-Peteiro M, Ucieda-Somoza R, et al. Glycated albumin, a precursor of advanced glycation end-products, upregulates NADPH oxidase and enhances oxidative stress in human endothelial cells: molecular correlate of diabetic vasculopathy. Diabetes Metab Res Rev 2010;26:550.

22.Zhao Z, Zhao C, Zhang XH, et al. Advanced glycation end products inhibit glucose-stimulated insulin secretion through nitric oxidedependent inhibition of cytochrome c oxidase and adenosine triphosphate synthesis, Endocrinology 2009;150(6):2569.

23.Wu G, Haynes TE, Yan W, et al. Presence of glutamine: fructose-6 phosphate amido transferase for glucosamine-6- phosphate synthesis in endotelial cells: effects of hyperglycaemic and glutamine. Diabetologia 2001;44:196.

24.Brownlee M. The pathobiology of diabetic complications: a unifying mechanism. Diabetes 2005;54:1615.

25.Du X, Matsumura T, Edelstein D, et al. Inhibition of GAPDH activity by poly (ADP-ribose) polymerase activates three major pathways of hyperglycemic danage in endothelial cells. J Clin Invest 2003;112:1049.

26.Nieuwdorp M, van Haeften TW, Gouverneur MC, et al. Loss of endothelial glycocalyx during acute hyperglycemic coincides with endothelial dysfunction and coagulation activation in vivo. Diabetes 2006;55:480.

27.Steinberg HO, Chaker H, Leaming R, et al. Obesity/insulin resistance is associated with endothelial dysfunction. Implications for the syndrome of insulin resistance. J Clin Invest 1996;97(11):2601.

28. Meigs JB, Hu, FB, Rifai N, et al. Biomarkers of endothelia dysfunction and risk of type 2 diabetes mellitus. JAMA 2004;291(16):1978.

29.Song Y, Manson JE, Tinker L, et al. Circulating levels of endothelial adhesión molecules and risk of diabetes in an etnically diverse cohort of women. Diabetes 2007;56(7):1898.

30.Tesauro M, Rizza S, Iantorno M, et al. Vascular, metabolic, and inflammatory abnormalities in nornoglycemic offspring of patients with type 2 diabetes mellitus. Mebolism 2007;56(3):413.

31.Gogg S, Smith U, Jansson PA. Increased MAPK activation and impaired insulin signaling in subcutaneous microvascular endothelial cells in type 2 diabetes: the role of endothelin-1. Diabetes 2009;58: 2238.

32.Calderón V, R. Disfunción endotelial. Diagnóstico 2012;51(1): 21

33.Wang XL, Zhang $\mathbf{L}$, Youker K, et al. Free fatty acids inhibit insulin signaling-stimulated endothelial nitric oxide synthase activation through upregulating PTEN or inhibiting Akt Kinase. Diabetes 2006;55:2301.

34.Mathew M, Tay E, Cusi K. Elevated plasma free fatty acids increase cardiovascular risk by inducing plasma biomarkers of endothelial activation, myeloperoxidase and PAI-1 in healthy subjects. Cardiovasc Diabeto 2010;19:1186

Contribución de autoría: CDB participó en la recolección de datos, revisión crítica del artículo y en la aprobación de la versión final. CDT participó en la recolección de datos, redacción del manuscrito, en la revisión crítica del artículo y en la aprobación final. LDT participó la recolección de datos, diseño del artículo, revisión crítica del artículo y en la aprobación de la versión final.

Conflicto de interés: Los autores declaran no tener conflictos de interés en relación a los contenidos de este documento.

Financiamiento: Autofinanciado.

Citar como: Delgado-Butron C, Delgado-Torres C, Delgado-Torres L. Diabetes Mellitus y Disfunción Endotelial. Diagnóstico (Lima). 2020;59(3):154-161.

DOI: 10.33734/diagnostico.v59i3.239

Correspondencia: Leonidas Delgado Torres. Correo electrónico: leonidadt@hotmail.com 